\title{
The Hubbard model in the canonical formulation
}

\author{
Sebastian Burri, Urs Wenger* \\ Albert Einstein Center for Fundamental Physics \\ Institute for Theoretical Physics \\ University of Bern \\ Sidlerstrasse 5 \\ CH-3012 Bern \\ Switzerland \\ E-mail: burriditp.unibe.ch, wengerditp.unibe.ch
}

\begin{abstract}
We describe non-relativistic fermions on the lattice (Hubbard model) in the canonical formulation using transfer matrices in fixed fermion number sectors such that the partition function becomes fully factorized in time. By analytically integrating out the auxiliary Hubbard-Stratanovich field due to the four-fermion interaction, we express the system in terms of discrete, local fermion occupation numbers which are the only remaining degrees of freedom. We show the close relation to the fermion loop and the fermion bag formulation. One can prove that in $1+1$ dimension the fermion sign problem is absent. Finally, we construct improved estimators for the ground state energy, 2-point functions, and for the chemical potential.
\end{abstract}

37th International Symposium on Lattice Field Theory - Lattice2019

16-22 June 2019

Wuhan, China

${ }^{*}$ Speaker. 


\section{Motivation}

The sign problem at finite fermion density can be understood as a manifestation of huge cancellations between different states which contribute to the partition function in a given basis. Obviously, all states are always present in the grand-canonical partition function $Z(\mu, T)$ for any values of the chemical potential $\mu$ and temperature $T$. However, only some states are relevant and important for the physics at specific values of $\mu$ and $T$ and contribute significantly to $Z(\mu, T)$, while all other states either have negligible weights or need to cancel out. A prominent example is given by the so-called Silver Blaze property of physical systems: certain physical states are completely invisible in the physical properties of the system up to the critical chemical potential $\mu_{c}$, when they suddenly become the main physical degrees of freedom driving the physics.

In the canonical formulation, fewer cancellations are necessary to describe the same physics, as compared to the grand-canonical formulation. This becomes clear by considering the dimension of the Fock space. Consider for example a system specified by the Hamiltonian $\mathcal{H}(\mu)$ and its grand-canonical partition function

$$
Z_{\mathrm{GC}}(\mu, T)=\operatorname{Tr}\left[e^{-\mathcal{H}(\mu) / T}\right]=\operatorname{Tr} \prod_{t} \mathcal{T}_{t}(\mu),
$$

where in the last step we assumed a discretized time and a Trotter decomposition, such that $\mathcal{T}_{t}(\mu)$ are transfer matrices at fixed time indices $t$. In the canonical formulation, the partition function reads

$$
Z_{\mathrm{C}}\left(N_{f}, T\right)=\operatorname{Tr}_{N_{f}}\left[e^{-\mathcal{H} / T}\right]=\operatorname{Tr} \prod_{t} \mathcal{T}_{t}^{\left(N_{f}\right)},
$$

where the trace is now restricted to states with a specific fermion number $N_{f}$. As a consequence, in the canonical formulation certain cancellations are more obvious than in the grand-canonical one. In QCD, for example, the canonical partition functions vanish for the number of quarks $N_{Q} \neq 0$ mod 3 due to the transformation properties of the Fock states, while in the grand-canonical formulation the cancellation is not obvious at all. Similarly, in the $N_{f}=1$ Schwinger model it is immediately obvious that in the canonical formulation only the states in the zero-charge sector are physical, because the contributions from the canonical states in the other sectors cancel due to the global $\mathrm{U}(1)$ symmetry, while in the grand-canonical formulation the cancellation is not explicit.

The canonical transfer matrices can be constructed for a large class of quantum field theories. In some supersymmetric Yang-Mills gauge theories, for example, the canonical formulation has been shown to lead to a solution of the fermion sign problem [1,2], based on the construction of the transfer matrices in and the close connection of the canonical formulation with the (dual) fermion loop or worldline formulation [3]. This, in turn, allows for the construction of efficient simulation algorithms, such as fermion bag $[4,5]$ and fermion worm algorithms [6]. Based on the construction of the canonical transfer matrices in QCD [7], one can show that the sign problem is absent in the strong coupling and heavy-dense limit, even though it is very severe just away from it. Nevertheless, in the related 3-state Potts model the sign problem at finite density can be solved by defining clusters with fixed fermion numbers in the canonical formulation [8]. In this context, it is worthwhile to note that the fermionic degrees of freedom in the canonical formulation are expressed in terms of local fermion occupation numbers $n_{x}=0,1$, and the only fermionic degrees of freedom are discrete index sets. 
One can also construct improved estimators for fermionic correlation functions using the canonical transfer matrices, potentially allowing for local (multi-level) update schemes with exponential improvement of the signal-to-noise ratio, or even for the direct Monte-Carlo sampling of the correlation function. In some cases, the canonical formulation makes it possible to integrate out explicitly the (auxiliary) bosonic degrees of freedom, such as the Hubbard-Stratanovich field in the Hubbard model, thereby solving the fermion sign problem explicitly in $d=1+1$ dimension.

In these proceedings, we consider the specific example of the Hubbard model and demonstrate in detail the connections and constructions mentioned above. We note that some of these ideas have been considered in the Hubbard model some time ago in $[9,10,11,12]$.

\section{Canonical formulation of the Hubbard model}

Let us consider the Hamiltonian for the Hubbard model,

$$
\mathcal{H}(\mu)=-\sum_{\langle x, y\rangle, \sigma} t_{\sigma} \hat{c}_{x, \sigma}^{\dagger} \hat{c}_{y, \sigma}+\sum_{x, \sigma} \mu_{\sigma} N_{x, \sigma}+U \sum_{x} N_{x, \uparrow} N_{x, \downarrow}
$$

where $\sigma=\downarrow, \uparrow$ denotes two fermion species, e.g., with spin up or down, $c_{x, \sigma}^{\dagger}$ and $c_{x, \sigma}$ are the creation and annihilation operators for the corresponding fermion at position $x$, while $\langle x, y\rangle$ denotes the nearest neighbours and $t_{\sigma}$ the hopping parameter. The four-fermion interaction between the fermions is parametrized by the local particle number $N_{x, \sigma}=\hat{c}_{x, \sigma}^{\dagger} \hat{c}_{x, \sigma}$ and the coupling strength $U$, while the total particle number is tuned through the chemical potentials $\mu_{\sigma}$.

The grand-canonical partition function can now be written as

$$
Z_{\mathrm{GC}}(\mu)=\operatorname{Tr}\left[e^{-\mathcal{H}(\mu) / T}\right]=\sum_{\left\{N_{\sigma}\right\}} e^{-\sum_{\sigma} N_{\sigma} \mu_{\sigma} / T} \cdot Z_{C}\left(\left\{N_{\sigma}\right\}\right)
$$

where in the last step we perform a fugacity expansion in order to express the grand-canonical partition function in terms of the canonical ones, i.e., $Z_{C}\left(\left\{N_{\sigma}\right\}\right)=\operatorname{Tr} \prod_{t} \mathcal{T}_{t}^{\left(\left\{N_{\sigma}\right\}\right)}$. Note that the canonical transfer matrices are now restricted to states with fixed total fermion number $N_{\sigma}=\sum_{x} N_{x, \sigma}$.

In order to investigate the model nonperturbatively using Monte Carlo simulations, one usually performs a Trotter decomposition and introduces a coherent state representation for the fermionic degrees of freedom, eventually yielding the partition function in terms of a path integral over Grassmann-valued fields $\psi^{\dagger}$ and $\psi$,

$$
Z_{\mathrm{GC}}(\mu)=\int \mathcal{D} \psi^{\dagger} \mathcal{D} \psi e^{-S\left[\psi^{\dagger}, \psi ; \mu\right]}
$$

with Euclidean action

$$
S\left[\psi^{\dagger}, \psi ; \mu\right]=\sum_{\sigma} \psi_{\sigma}^{\dagger} \nabla_{t} \psi_{\sigma}+H\left[\psi^{\dagger}, \psi ; \mu\right],
$$

where the Hamilton function $H$ describes the spatial hoppings of the Grassmann fields as well as the four-fermion interaction. This interaction can be bilinearized by a Hubbard-Stratonovich (HS) transformation using the HS field $\phi$ leading to

$$
Z_{\mathrm{GC}}(\mu)=\int \mathcal{D} \psi^{\dagger} \mathcal{D} \psi \mathcal{D} \phi \rho[\phi] e^{-\sum_{\sigma} S\left[\psi_{\sigma}^{\dagger}, \psi_{\sigma}, \phi ; \mu_{\sigma}\right]},
$$


with the action $S\left[\psi_{\sigma}^{\dagger}, \psi_{\sigma}, \phi ; \mu_{\sigma}\right]=\psi_{\sigma}^{\dagger} M\left[\phi ; \mu_{\sigma}\right] \psi_{\sigma}$ now being a bilinear form in the fermion fields $\psi_{\sigma}^{\dagger}, \psi_{\sigma}$ involving the fermion matrix $M$, and $\rho[\phi]$ being the Boltzmann factor for the HS field $\phi$. The fermion fields can now be integrated out, yielding the determinant of $M$,

$$
Z_{\mathrm{GC}}(\mu)=\int \mathcal{D} \phi \rho[\phi] \prod_{\sigma} \operatorname{det} M\left[\phi ; \mu_{\sigma}\right]
$$

The HS transformation can be chosen in such a way, cf. e.g. [13], that the fermion matrix has the structure

$$
M\left[\phi ; \mu_{\sigma}\right]=\left(\begin{array}{cllc}
B & 0 & \ldots & \pm e^{\mu_{\sigma}} C\left(\phi_{L_{t}-1}\right) \\
-e^{\mu_{\sigma}} C\left(\phi_{0}\right) & B & \ldots & 0 \\
\vdots & \ddots & \ddots & \vdots \\
0 & \ldots & -e^{\mu_{\sigma}} C\left(\phi_{L_{t}-2}\right) & B
\end{array}\right)
$$

where $B$ and $C$ are matrices of size $L_{s} \times L_{s}$, and $L_{s}$ and $L_{t}$ are the number of spatial and temporal lattice points. Note that while $B$ is a constant matrix, $C=C\left(\phi_{t}\right)$ only depends on the HS fields on time slice $t$ and can be chosen diagonal. The determinant of $M$ can be dimensionally reduced to [9]

$$
\operatorname{det} M\left[\phi ; \mu_{\sigma}\right]=\operatorname{det} B^{L_{t}} \cdot \operatorname{det}\left(1 \mp e^{L_{t} \mu_{\sigma}} \mathcal{T}[\phi]\right)
$$

where $\mathcal{T}[\phi]=B^{-1} C\left(\phi_{L_{t}-1}\right) \cdot \ldots \cdot B^{-1} C\left(\phi_{0}\right)$. The fugacity expansion of this object yields the canonical determinants $\operatorname{det} M_{N_{\sigma}}[\phi]$ as the coefficients of the corresponding fugacity terms. They can be expressed as a sum over the principal minors $\operatorname{det} \mathcal{T}{ }^{X X}$ of order $N_{\sigma}$,

$$
\operatorname{det} M_{N_{\sigma}}[\phi]=\sum_{J} \operatorname{det} \mathcal{T}^{\mathcal{X X}}[\phi]=\operatorname{Tr}\left[\prod_{t} \mathcal{T}_{t}^{\left(N_{\sigma}\right)}\left[\phi_{t}\right]\right],
$$

where the index sets $J \subset\left\{1, \ldots, L_{s}\right\}$ of size $N_{\sigma}=|J|$ essentially label the states of the fermion Fock space and therefore allow to identify the sum as the trace over the product of transfer matrices acting in the Fock space of states with fixed fermion number $N_{\sigma}$. Indeed, in sector $N_{\sigma}$ the number of states $N_{\text {states }}=\left(\begin{array}{c}L_{s} \\ N_{\sigma}\end{array}\right)$ is equal to the number of principal minors of order $N_{\sigma}$. At half-filling, this number grows exponentially with $L_{s}$, however, it can efficiently be evaluated stochastically with Monte Carlo methods, as for example in [1,2].

\section{Transfer matrices of the Hubbard model}

Using eq. (2.1) we can now identify the transfer matrices and write down explicit expressions for them. To this end we recall the Cauchy-Binet formula

$$
\operatorname{det}(A \cdot B)^{\chi_{K}}=\operatorname{det} A^{\chi \chi \chi} \cdot \operatorname{det} B^{\text {\K }}
$$

and factorize $\mathcal{T}[\phi]$ into the product of transfer matrices $\mathcal{T}_{t}^{\left(N_{\sigma}\right)}\left[\phi_{t}\right]$, which are hence given by

$$
\left(\mathcal{T}_{t}^{\left(N_{\sigma}\right)}\right)_{I K}=\operatorname{det} B \cdot \operatorname{det}\left[B^{-1} \cdot C\left(\phi_{t}\right)\right]^{\lambda_{K}}=\operatorname{det} B \cdot \operatorname{det}\left(B^{-1}\right)^{\lambda \chi} \cdot \operatorname{det} C\left(\phi_{t}\right)^{X_{K}},
$$

where $|I|=|J|=|K|=N_{\sigma}$. Moreover, using the complementary cofactor we have

$$
\operatorname{det} B \cdot \operatorname{det}\left(B^{-1}\right)^{X X}=(-1)^{p(I, J)} \operatorname{det} B^{I J},
$$


where $p(I, J)=\sum_{i}\left(I_{i}+J_{i}\right)$. Since the HS transformation can be chosen such that $C\left(\phi_{t}\right)$ is diagonal, we find

$$
\operatorname{det} C\left(\phi_{t}\right)^{x K}=\delta_{J K} \prod_{x \notin J} \phi_{x, t}
$$

and the HS field can be integrated out site by site,

$$
\int d \phi_{x, t} \rho\left(\phi_{x, t}\right) \phi_{x, t}^{\sum_{\sigma} \delta_{x \notin J \sigma}} \equiv w_{x, t}= \begin{cases}w_{2}, & \text { if } x \notin J^{\uparrow}, x \notin J^{\downarrow}, \\ w_{1}, & \text { else, } \\ w_{0}, & \text { if } x \in J^{\uparrow}, x \in J^{\downarrow},\end{cases}
$$

where $w_{i}>0$. Finally, with $\prod_{x} w_{x, t} \equiv W\left(\left\{J_{t}^{\sigma}\right\}\right)$ we have the canonical partition function of the Hubbard model expressed in fully factorized form,

$$
Z_{C}\left(\left\{N_{\sigma}\right\}\right)=\sum_{\left\{J_{t}^{\sigma}\right\}} \prod_{t}\left(\prod_{\sigma} \operatorname{det} B^{J_{t-1}^{\sigma} J_{t}^{\sigma}}\right) W\left(\left\{J_{t}^{\sigma}\right\}\right), \quad\left|J_{t}^{\sigma}\right|=N_{\sigma},
$$

with the only dynamical degrees of freedom being the index sets $\left\{J_{t}^{\sigma}\right\}$ on each time slice. The representation in eq. (3.1) exposes the relation to the fermion loop formulation. Interpreting the spatial indices in the index sets $J_{t}^{\sigma}$ as labelling the positions of the $N_{\sigma}$ fermions where they hop in time, one can identify the $N_{\sigma}$ fermion loops running around the lattice in temporal direction, cf. Figure 1 for an illustration with $N_{\sigma}=$ 2 in $d=1$ spatial dimension. The factors $\operatorname{det} B^{J_{t-1}^{\sigma} J_{t}^{\sigma}}$ collect all the contributions from different fermion loop configurations on time slice $t$ within the shaded regions. They can therefore be interpreted as "fermion bags". It turns out that in $d=1$ spatial dimensions

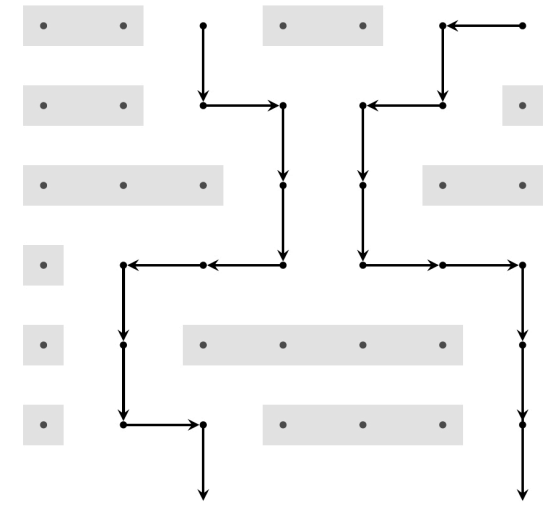

Figure 1: Illustration of a fermion loop configuration and the corresponding index sets $J_{t}$. the contributions $\operatorname{det} B^{I J}$ can be calculated analytically through the recursion relation [14]

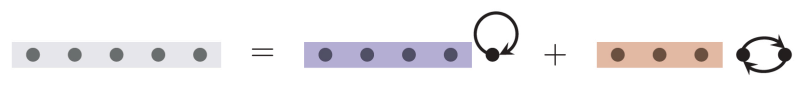

and for open b.c. one can prove that $\operatorname{det} B^{I J} \geq 0$, i.e., there is no sign problem. For periodic b.c. there is no sign problem either, because in the thermodynamic limit, the partition functions become independent of the boundary conditions.

\section{Improved estimators}

Having the canonical partition function in its fully factorized form, we can construct improved estimators for various observables. The energy of the ground state, for example, is defined by $E_{0}=-\lim _{\beta \rightarrow \infty} \partial_{\beta} \ln Z_{C}(\beta)$, which on the lattice becomes

$$
E_{0}=\lim _{L_{t} \rightarrow \infty} \ln \frac{Z_{C}\left(L_{t}\right)}{Z_{C}\left(L_{t}+1\right)} .
$$



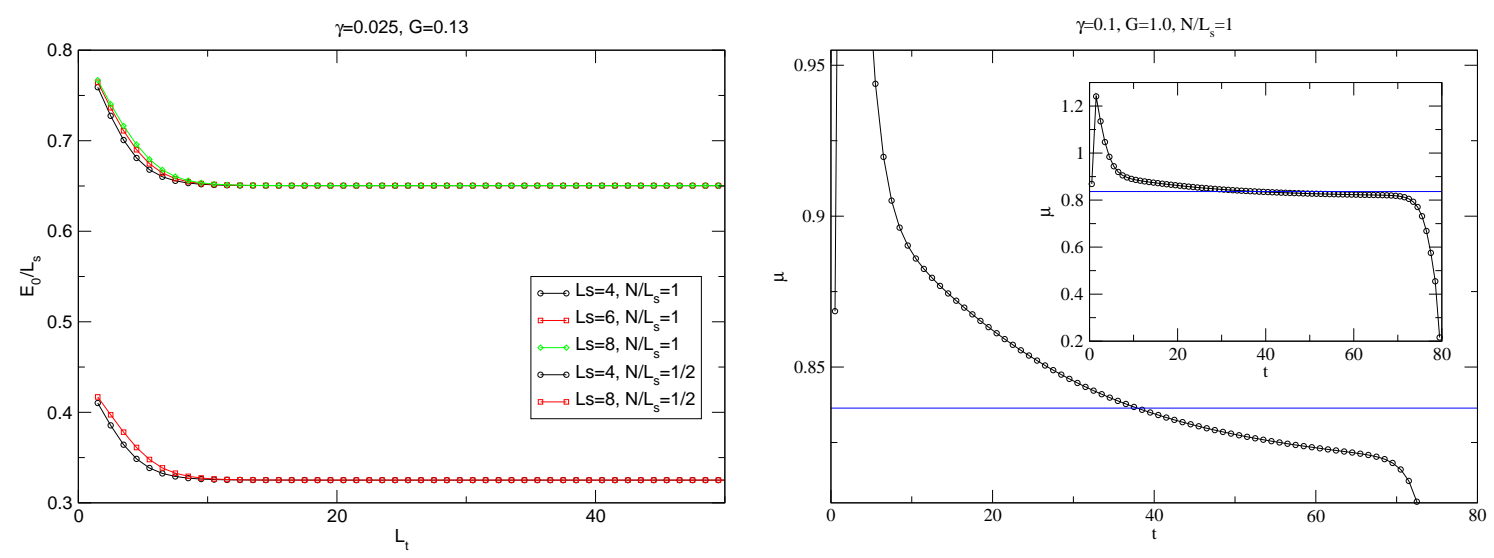

Figure 2: Left plot: Logarithm of the partition function ratios $Z_{C}\left(L_{t}\right) / Z_{C}\left(L_{t}+1\right)$ as a function of $L_{t}$ which yields the ground state energy $E_{0}$ in the limit $L_{t} \rightarrow \infty$. Right plot: Chemical potential from eq. (4.2) as a function of $t$. The blue line is the value obtained via eq. (4.1) from the ratio $Z_{C}\left(n, n_{s}\right) / Z_{C}\left(n+1, n_{s}+1\right)$.

Since our formulation is factorized in time, we can write the ratio as an observable calculated in the ensemble defining $Z_{C}\left(L_{t}+1\right)$,

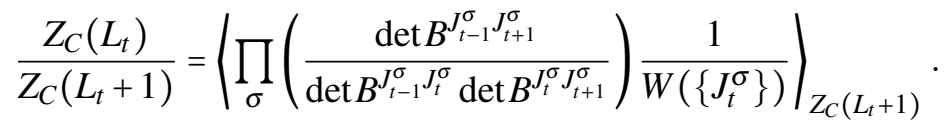

In the left plot of Figure 2 we show the logarithm of this ratio (normalized by the volume $L_{s}$ ) as a function of the inverse temperature $1 / T=L_{t}$ for two different fermion densities $\rho=N / L_{s}=$ $\left(N_{\uparrow}+N_{\downarrow}\right) / L_{s}=1$ (half-filling) and $1 / 2$ (quarter-filling) for specific values of the hopping $t_{\uparrow}=t_{\downarrow}$ and the four-fermion coupling $U$ (parametrized by $\gamma$ and $G$ ). As another example we consider the chemical potentials $\mu_{(s)}$ defined as $\mu_{(s)}=\partial_{\rho_{(s)}} F\left(\rho_{(s)}\right)$ with $\rho_{(s)}=\left(N_{\uparrow} \pm N_{\downarrow}\right) / L_{s}$ which on the lattice can be written as, e.g.,

$$
\mu=\frac{F\left(n+2, n_{s}\right)-F\left(n, n_{s}\right)}{2}=-\frac{1}{2 L_{t}} \ln \left(\frac{Z_{C}\left(n+2, n_{s}\right)}{Z_{C}\left(n, n_{s}\right)}\right) .
$$

Now we define the partition function for the fermionic 2-pt. function of, e.g., an up-spin fermion in the background of $\left\{N_{\sigma}\right\}$ fermions,

$$
Z_{t}^{2 \text {-pt., }}\left(\left\{N_{\sigma}\right\}\right)=\operatorname{Tr}\left[P^{\dagger} \prod_{t^{\prime}<t} \mathcal{T}_{t^{\prime}}^{\left\{N_{\uparrow}+1, N_{\downarrow}\right\}} P \cdot \prod_{t^{\prime} \geq t} \mathcal{T}_{t^{\prime}}^{\left\{N_{\sigma}\right\}}\right]=\left\langle\left\langle\psi_{0}^{\dagger} \psi_{t}\right\rangle\right\rangle_{\left\{N_{\sigma}\right\}},
$$

where $P^{\dagger}(P)$ create (annihilate) an up-spin fermion at time $t^{\prime}=0\left(t^{\prime}=t\right)$. Then we can write the chemical potential as a telescopic product of ratios of 2-pt. functions,

$$
e^{-2 \mu / T}=\frac{Z_{0}^{2-\text { pt. }, \uparrow}}{Z\left(n, n_{s}\right)} \cdot \frac{Z_{1}^{2-\text { pt. }, \uparrow}}{Z_{0}^{2-\text { pt. }, \uparrow}} \cdot \cdots \cdot \frac{Z\left(n+1, n_{s}+1\right)}{Z_{L t-1}^{2-\text { pt. } \uparrow}} \times \frac{Z_{0}^{2-\text { pt. }, \downarrow}}{Z\left(n+1, n_{s}+1\right)} \cdot \frac{Z_{1}^{2-\text { pt. }, \downarrow}}{Z_{0}^{2-\text { pt. }, \downarrow}} \cdot \ldots \cdot \frac{Z\left(n+2, n_{s}\right)}{Z_{L_{t}-1}^{2-\text { t. } \downarrow}} .
$$

Each ratio can in turn be written as an expectation value, e.g.,

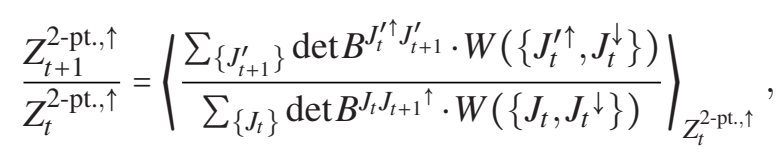


where $\left|J_{t+1}^{\prime}\right|=\left|J_{t}\right|+1=N_{\uparrow}+1$, and the chemical potential can be calculated from the logarithmic sum of the corresponding observables. In the right plot of Figure 2 we show the chemical potential for a single fermion obtained in this way. The shape of the curve depends very much on the operators $P^{\dagger}, P$, i.e., the wave function used to create and annihilate the fermion.

\section{References}

[1] G. Bergner, H. Liu and U. Wenger, Canonical simulations of supersymmetric SU(N) Yang-Mills quantum mechanics, PoS LATTICE2015 (2016) 241, [1509.01446].

[2] G. Bergner, H. Liu and U. Wenger, A local update algorithm for supersymmetric Yang-Mills quantum mechanics, PoS LATTICE2016 (2016) 395, [1612.04291].

[3] K. Steinhauer and U. Wenger, Loop formulation of supersymmetric Yang-Mills quantum mechanics, JHEP 12 (2014) 044, [1410 . 0235].

[4] S. Chandrasekharan, The Fermion bag approach to lattice field theories, Phys. Rev. D82 (2010) 025007, [0 910.5736 ].

[5] S. Chandrasekharan, Fermion Bag Approach to Fermion Sign Problems, Eur. Phys. J. A49 (2013) 90, [1304.4900].

[6] U. Wenger, Efficient simulation of relativistic fermions via vertex models, Phys. Rev. D80 (2009) 071503, [0812.3565].

[7] A. Alexandru and U. Wenger, QCD at non-zero density and canonical partition functions with Wilson fermions, Phys. Rev. D83 (2011) 034502, [1009.2197].

[8] A. Alexandru, G. Bergner, D. Schaich and U. Wenger, Solution of the sign problem in the Potts model at fixed fermion number, Phys. Rev. D97 (2018) 114503, [1712.07585].

[9] R. Blankenbecler, D. J. Scalapino and R. L. Sugar, Monte carlo calculations of coupled boson-fermion systems. I, Phys. Rev. D 24 (1981) 2278-2286.

[10] J. E. Hirsch, R. L. Sugar, D. J. Scalapino and R. Blankenbecler, Monte Carlo Simulations of One-dimensional Fermion Systems, Phys. Rev. B26 (1982) 5033-5055.

[11] J. E. Hirsch, Connection between world-line and determinantal functional-integral formulations of the hubbard model, Phys. Rev. B 34 (1986) 3216-3220.

[12] S. R. White, D. J. Scalapino, R. L. Sugar, E. Y. Loh, J. E. Gubernatis and R. T. Scalettar, Numerical study of the two-dimensional hubbard model, Phys. Rev. B 40 (1989) 506-516.

[13] A. Alexandru, P. F. Bedaque and N. C. Warrington, Spin polarized nonrelativistic fermions in $1+1$ dimensions, Phys. Rev. D98 (2018) 054514, [1805.00125].

[14] M. G. Endres, Lattice theory for nonrelativistic fermions in one spatial dimension, Phys. Rev. $\mathbf{A 8 5}$ (2012) 063624, [1204.6182]. 